\title{
Transient-grating single-shot supercontinuum spectral interferometry (TG-SSSI)
}

\author{
S. W. Hancock, S. Zahedpour, H. M. Milchberg \\ Institute for Research in Electronics and Applied Physics, University of Maryland, College Park, \\ Maryland, 20742, USA
}

\begin{abstract}
We present a technique for the single-shot measurement of the space- and time-resolved spatiotemporal amplitude and phase of an ultrashort laser pulse. The method, transient-grating single-shot supercontinuum spectral interferometry (TGSSSI), is demonstrated by the space-time imaging of short pulses carrying spatiotemporal optical vortices (STOVs). TG-SSSI is well-suited for characterizing ultrashort laser pulses that contain singularities associated with spin/orbital angular momentum or polarization.
\end{abstract}

\section{Introduction}

The need to characterize ultrashort laser pulses has spawned a large and increasing number of single-shot techniques including autocorrelation [1], multiple versions of frequency resolved optical gating (FROG) [2-6], spectral phase interferometry for direct electric-field reconstruction (SPIDER) and related methods [7-12], STRIPED FISH [13], d-scan [14], plus single-shot supercontinuum spectral interferometry (SSSI) [15-17] and other spectral interferometry methods $[18,19]$. While the basic FROG and SPIDER techniques extract only the space-independent temporal amplitude and phase, more complicated techniques [12-14] have recovered the spatiotemporal phase and amplitude of a laser pulse in a single-shot, albeit only with simple features such as pulsefront tilt. STRIPED-FISH [13] and d-scan [14] methods use iterative algorithms which, to our knowledge, have not been shown to converge for complicated structured light containing singularities, and SEA-SPIDER requires ancillary assumptions in determining the timing of spatial slices [12]. While SSSI does not recover the spatiotemporal phase of a pulse, it does recover the spatiotemporal pulse envelope, which has enabled measurement of ionization rates and ultrafast plasma evolution [20], electronic, vibrational and rotational nonlinearities $[21,22]$, as well as nonlinear refractive indices and pulse front tilt [23].

In this paper, we present a new method that can measure, in a single-shot, the spatiotemporal phase and amplitude of an ultrafast laser pulse. It was developed for recent measurements [24] of pulses embedded with spatiotemporal optical vortices (STOVs) [25] and is well-suited for characterizing ultrashort laser pulses that contain singularities associated with spin/orbital angular momentum (SAM/OAM) [26-28] or polarization [29].

\section{Experimental Setup}

We first briefly review SSSI by examining three of the beams in Fig. 1: the "structured pulse" $E_{S}$ which we want to measure, the reference pulse $E_{r e f}$, and the probe pulse $E_{p r}$. Here, the structured pulse has spatiotemporal phase and amplitude imposed by the zero dispersion $4 f$ pulse shaper [30-32] in the lower left of the figure. The reference and probe supercontinuum (SC) pulses are generated upstream of Fig. 1 by filamentation in a $2 \mathrm{~atm} \mathrm{SF}_{6}$ cell followed by a Michelson interferometer (not shown), with $E_{\text {ref }}$ leading 


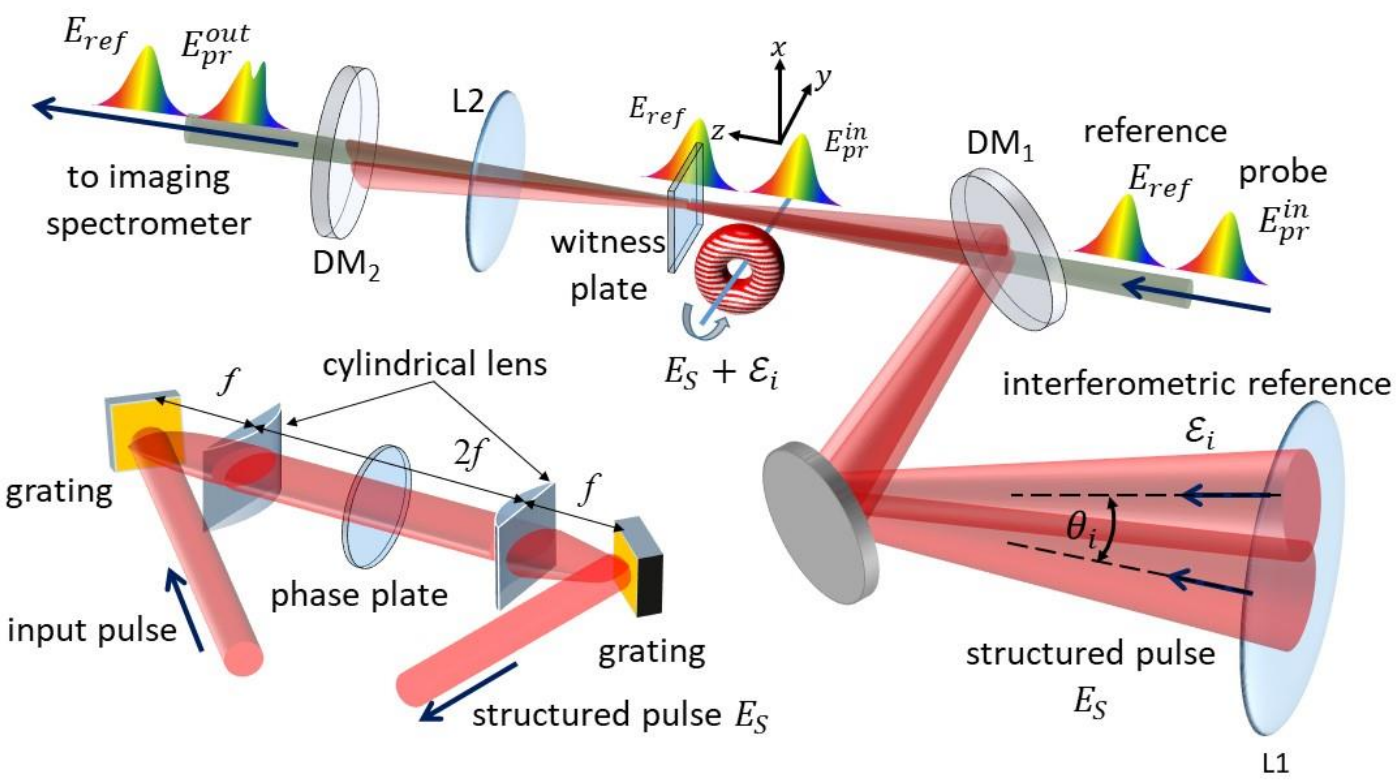

Fig. 1. Setup for transient grating single-shot spectral interferometry (TG-SSSI). The structured pulse $E_{S}$ and the interferometric reference pulse $\varepsilon_{i}$ cross at angle $\theta_{w}$ at the focus of lens L1 in a fused silica witness plate $\left(\theta_{i}\right.$ in air), where a transient grating is formed. The grating is probed by a supercontinuum (SC) probe $E_{p r}^{i n}$, preceded $\sim 2$ ps earlier by a reference SC pulse $E_{r e f}$. Imaged by L2, $E_{r e f}$ and $E_{p r}^{\text {out }}$ interfere at an imaging spectrometer and the interferogram is analyzed in the Fourier domain, yielding a single-shot time and space resolved image of amplitude and phase of $E_{S}$. Bottom left: $4 f$ pulse shaper [30-32] for generating spatiotemporally structured pulses $E_{S}$, here STOVs $[24,33,34]$ imposed on a $50 \mathrm{fs}, \lambda=800 \mathrm{~nm}$ input pulse. The STOV phase windings are imposed by $l= \pm 1$, and $l=-2$ spiral phase plates in the Fourier plane of the pulse shaper. The phase plates are etched on fused silica and have 16 levels (steps) every $2 \pi$.

$E_{p r}$ by $\sim 2$ ps. The transient amplitude of $E_{S}$ is measured via the phase modulation it induces in a spatially and temporally overlapped SC probe pulse $E_{p r}$ in an instantaneous Kerr "witness plate", here a thin (100$500 \mu \mathrm{m})$ fused silica window. The resulting spatio-spectral phase shift $\Delta \varphi(x, \omega)$ imposed on the probe is extracted from interfering $E_{p r}^{\text {out }} \sim \chi^{(3)} E_{S} E_{S}^{*} E_{p r}^{\text {in }}$ with $E_{r e f}$ in an imaging spectrometer. Here, $E_{p r}^{\text {in }}$ and $E_{p r}^{o u t}$ are the probe fields entering and exiting the witness plate, $\chi^{(3)}$ is the fused silica nonlinear susceptibility, and $x$ is position within a 1D transverse spatial slice through the pump pulse at the witness plate (axes shown in Fig. 1). Fourier analysis of the extracted $\Delta \varphi(x, \omega)$ [20] then determines the spatiotemporal phase shift $\Delta \phi(x, \tau) \propto\left|E_{S}(x, \tau)\right|^{2} \propto I_{S}(x, \tau)$, yielding the 1D space + time spatio-temporal intensity envelope $I_{S}$.

Measurement of the spatiotemporal phase of $E_{S}$ is enabled by the addition of an interferometric reference pulse $\varepsilon_{i}$, which is crossed with $E_{S}$ at a small angle $\theta_{i}\left(\theta_{w}=3.15^{\circ}\right.$ in the witness plate). This forms a nonlinear transient refractive index grating, where $\varepsilon_{i}$ has the same center wavelength as $E_{S}$ but is bandpassed to be temporally longer. The transient grating (TG) is now the signal probed by SSSI (yielding the new method we call TG-SSSI), where the output probe pulse from the witness plate becomes $E_{p r}^{\text {out }} \propto \chi^{(3)} E_{S} \varepsilon_{i}^{*} E_{p r}^{\text {in }}$. The interference of $E_{p r}^{\text {out }}$ and $E_{r e f}$ in the imaging spectrometer then enables extraction of $\Delta \varphi(x, \omega)$, yielding $\Delta \phi(x, \tau)$ as before. We note that $\Delta \phi(x, \tau)$ is the envelope of $E_{S}$ modulated by the transient grating: $\Delta \phi(x, \tau) \propto I_{S}(x, \tau) f(x, \tau)$, where $f(x, \tau)=\cos \left(2 k_{w} x \sin \left(\theta_{w} / 2\right)+\Delta \Phi(x, \tau)\right)$ is the transient grating, $k_{w}=n_{0} k$ is the pump central wavenumber in the witness plate, $n_{0}=1.45$, and $\Delta \Phi(x, \tau)$ is the spatiotemporal phase of $E_{S}$. 
In the analysis of the 2D $\Delta \phi(x, \tau)$ images, $\Delta \Phi(x, \tau)$ is extracted using standard interferogram analysis techniques $[13,14]$, and $I_{S}(x, \tau)$ is extracted using a low pass image filter (suppressing the sidebands imposed by the transient grating). Due to group velocity mismatch in the witness plate between $E_{S}$ (centre wavelength $\left.\lambda_{0}=800 \mathrm{~nm}\right)$ and the SC probe $\left(\lambda_{p r}=600 \mathrm{~nm}\right)$, the extracted phase shift is smeared slightly in time by $\sim 4 \mathrm{fs}$ per $100 \mu \mathrm{m}$ of fused silica.

The laser used in the experiments is a $4 \mathrm{~mJ} /$ pulse, $50 \mathrm{fs}$ FWHM, $\lambda_{0}=800 \mathrm{~nm}, 1 \mathrm{kHz}$ Ti:Sapphire system. The beam is split 3 ways, with $\sim 100 \mu \mathrm{J}$ directed to SC generation (400$700 \mathrm{~nm})$ for $E_{p r}$ and $E_{r e f}$, and a portion of the rest for $E_{S}$ and $\varepsilon_{i}$, whose energies were controlled using $\lambda / 2$ plates and thin-film polarizers. The structured pulse $E_{S}$ was embedded with spatiotemporal phase windings by placing $l= \pm 1$ or $l=-2$ spiral phase plates at the Fourier plan of the $4 f$ pulse shaper [24].

As depicted in Fig. 1, the SC reference and probe pulses, $E_{r e f}$ and $E_{p r}^{i n}$, are combined collinearly with the pulse $E_{S}$ using dichroic mirror $\mathrm{DM}_{1}$, with $E_{S}, \varepsilon_{i}$, and $E_{p r}^{\text {in }}$ overlapping temporally in the witness plate, while $E_{\text {ref }}$ precedes them (by $2 \mathrm{ps}$ ). From the output face of the witness plate, $E_{\text {ref }}$ and $E_{p r}^{\text {out }}$ were magnified and relay imaged onto the spectrometer slit using high numerical aperture (NA) telescope with achromatic lenses. The large NA is necessary to collect the first order diffraction $(m= \pm 1)$ of $E_{p r}^{o u t}$ from the transient grating. It is important for the imaging lenses to be achromatic for the image at the spectrometer slit to be in focus for all SC wavelengths and to minimize spherical aberration, which could spatially offset the diffracted orders of $E_{p r}^{\text {out }}$ from the zero order. Background and signal data were collected at $40 \mathrm{~Hz}$ by placing

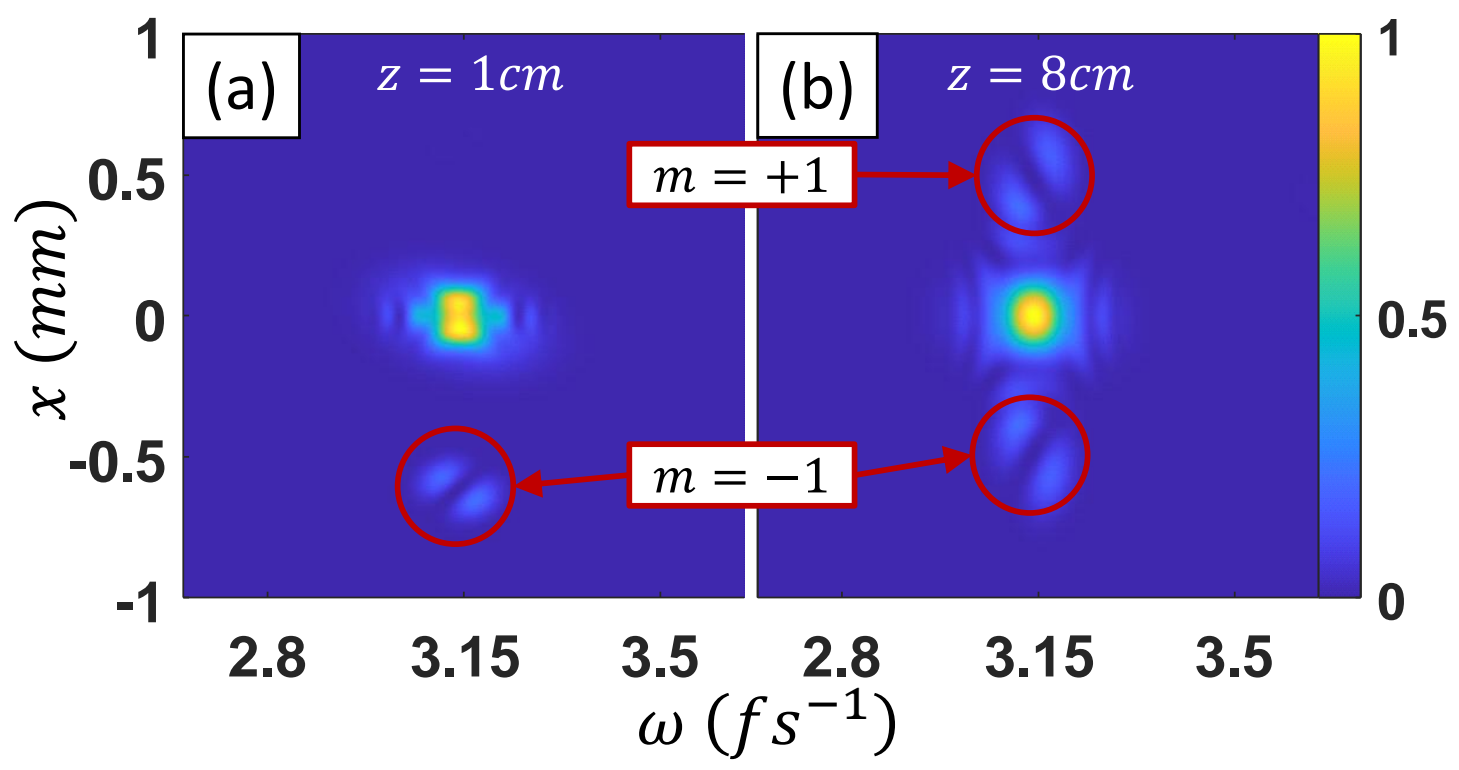

Fig. 2. Simulated spectrally-resolved scattering of supercontinuum probe pulse $E_{p r}$ from a transient nonlinear grating in a $500 \mu \mathrm{m}$ thick fused silica plate, generated by the interference of pulses $E_{S}$ and $\varepsilon_{i}$, where $z$ is distance from the output face of the plate. Plotted as $\left|\Delta E_{p r}\right|^{2}=\left|E_{p r}^{o u t}-E_{p r}^{i n}\right|^{2}$.(a) Bragg regime transient grating: $\theta_{w}=3.15^{\circ}$, grating period $\Lambda=10 \mu \mathrm{m}$, and $Q=13.0$. Here, the scattering is captured at $z=1 \mathrm{~cm}$ owing to the rapid escape of the single diffracted order $(m=-1)$ from the simulation window. (b) Raman-Nath regime transient grating: $\theta_{w}=0.31^{\circ}, \Lambda=100 \mu m$, and $Q=0.13$, showing $m= \pm 1$ diffraction orders. 
a chopper in the path of $E_{S}$, which allowed for the subtraction of the phase shift induced by $\varepsilon_{i}$ in the witness plate.

In principle, achromatic imaging of all diffracted orders precludes the need for detailed analysis of the diffraction. However, it is interesting to note that in our experiment, we observe only the zero order $(m=0)$ and the $m=-1$ order diffraction of the probe. To understand this, we assess whether probe diffraction is in the Bragg regime (one dominant diffraction peak) or in the RamanNath regime (multiple positive and negative diffraction orders) [35] by considering the dimensionless parameter $Q=2 \pi \lambda_{i} L / \Lambda^{2} \bar{n}$, where $\lambda_{i}$ is the vacuum wavelength of incident light, $\Lambda$ is the interference grating period, $\bar{n}$ is the mean refractive index, and $L$ is the grating thickness. From ref. [35], diffraction is in the Raman-Nath regime for $Q \leq 1$ and in the Bragg regime for $Q \gg 1$. Our TG-SSSI configuration (with a $l=500 \mu \mathrm{m}$ fused silica witness plate, $\Lambda\left(\theta_{w}=\right.$ $\left.3.15^{\circ}\right)=10 \mu \mathrm{m}, \quad n_{2}=2.5 \times 10^{-16} \mathrm{~cm}^{2} / \mathrm{W}$, and $\bar{n}=n_{0}+n_{0} n_{2} I=n_{0}+\Delta \phi_{T G} / k L$, where $\Delta \phi_{T G}$ is the modulated phase shift amplitude of the transient grating) gives $Q \cong 13.0$, which is in the Bragg regime. (Both $\Delta \phi_{T G}$ and $\Lambda$ are from our measurements.) This explains the observation of only one diffracted order.

This result is confirmed by simulations of scattering of $E_{p r}$ from the nonlinear grating formed by the interference of $E_{S}$ and $\varepsilon_{i}$. The simulation uses our implementation [36] of the unidirectional pulse propagation equation (UPPE) method [37], where all 3 beams intersect in the 500 $\mu \mathrm{m}$ thick fused silica plate (with $E_{S}$ and $\varepsilon_{i}$ crossing at angle $\theta_{w}$ and $E_{p r}^{i n}$ normal to the surface). The beam parameters are $E_{S}\left(\lambda=800 \mathrm{~nm}, 50 \mathrm{fs}\right.$ FWHM, $w_{0}=100 \mu \mathrm{m}, I_{S, \text { peak }}=28 \mathrm{GW} / \mathrm{cm}^{2}, l=+1$ STOV), $\varepsilon_{i}\left(\lambda=800 \mathrm{~nm}, 300 \mathrm{fs}\right.$ FWHM, $\left.w_{0}=300 \mu \mathrm{m}, I_{i, p e a k}=28 \mathrm{GW} / \mathrm{cm}^{2}, \mathrm{GDD}=0 \mathrm{fs}^{2}\right)$, and $E_{p r}^{i n}\left(\lambda=600 \mathrm{~nm}, \Delta \lambda=350 \mathrm{~nm}, 2.4 \mathrm{ps} \quad\right.$ FWHM $, \quad w_{0}=500 \mu \mathrm{m}, \quad$ GDD $=1200 \mathrm{fs}^{2}, \mathrm{TOD}=$ $200 \mathrm{fs}^{3}$ ). The output electric field is numerically propagated $4 \mathrm{~cm}$ beyond the witness plate in air and then $E_{S}$ and $\varepsilon_{i}$ are spectrally filtered out, leaving the field $E_{p r}^{o u t}$. Figure 2(a) shows simulation results of probe diffraction for conditions similar to our experimental parameters $\left(\theta_{w}=3.15^{\circ}\right.$ and $Q=13.0$ ), where only the $m=-1$ diffraction order is present (Bragg regime), agreeing with our experiments. The crossing angle for Fig. 2(b) was chosen to be $\theta_{w}=0.31^{\circ}$, giving $Q=0.13$, in the Raman-Nath regime, and the $m= \pm 1$ orders are present. We note that our current TG-SSSI setup could be adjusted to operate in the Raman- Nath regime by increasing the grating period $\Lambda$ (to $\Lambda \geq \sqrt{2 \pi \lambda_{i} L / \bar{n}}$ ), but increasing the intensity of $E_{S}$ or $\varepsilon_{i}$ to increase $n_{1}$ could result in nonnegligible plasma formation in the witness plate and refractive distortion of $E_{p r}$.

Figure 3(a) shows an example of a raw TG-SSSI interferogram frame recorded on the imaging spectrometer camera. Here, the pulse shaper generates $E_{S}$ as a $l=-2$ spatiotemporal optical vortex (STOV) pulse [24,25]. The vertical spectral fringe spacing is set by the Michelson-imposed time delay between the $E_{r e f}$ and $E_{p r}^{o u t}$ pulses. The 2D phase shift $\Delta \varphi(x, \omega)$ is extracted in the same way as with all other SSSI interferograms [14,15], yielding $\Delta \phi(x, \tau) \propto I_{S}(x, \tau) f(x, \tau)$, which is plotted in Fig. 3(b). Here, the horizontal fringes imposed by $f(x, \tau)$ show the time-dependent interference between $E_{S}$ and $\varepsilon_{i}$. The spatiotemporal pulse envelope is recovered by low pass image filtering of $\Delta \phi(x, \tau)$ to remove $f(x, \tau)$, yielding $I_{s}(x, \tau)$ in Fig. 3(c).

Extraction of the spatiotemporal phase $\Delta \Phi(x, \tau)$ is performed by Fourier analysis along $x$ [38],

$$
\Delta \Phi(x, \tau)=\arg \left(\mathcal{F}_{k}^{-1}\left\{\mathcal{F}_{x}\{\Delta \phi(x, \tau)\} \Theta(k)\right\}\right),
$$



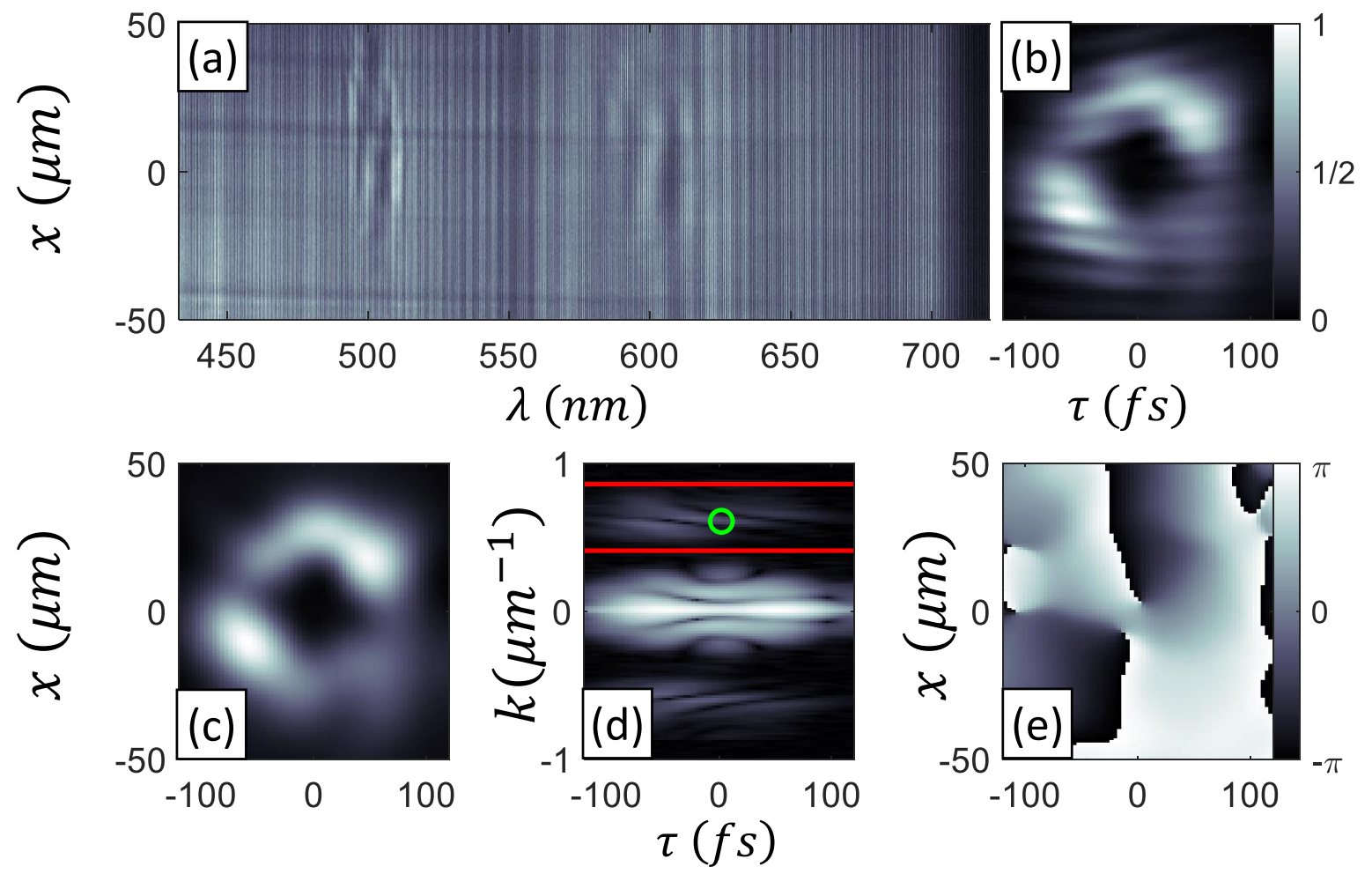

Fig. 3. Measurement of a $l=-2$ STOV-carrying pulse (interferometric reference $\varepsilon_{i}$ at 800nm and 10nm FWHM bandwidth); (a) Raw 1D space-resolved spectral interferogram; (b) extracted $\Delta \phi(x, \tau)$; (c) pulse envelope $I_{S}(x, \tau)$ from low pass filtering of $\Delta \phi(x, \tau) ;(\mathbf{d}) \log \left(\left|\mathcal{F}_{x}\{\Delta \phi(x, \tau)\}\right|+1\right)$. The red lines show the region to be spectrally windowed and the green circle identifies $\left(k_{c}, \tau_{c}\right)$ for frame averaging; (e) extracted spatiotemporal phase of the pulse, $\Delta \Phi(x, \tau)$.

where $\mathcal{F}_{x}\{\Delta \phi(x, \tau)\}=\Delta \tilde{\phi}(k, \tau)$ is the Fourier transform along $x, \mathcal{F}_{k}^{-1}\{\cdot\}$ is the inverse Fourier transform along $k, \Theta(k)$ is a sideband windowing and shifting $(k \rightarrow k-2 \pi / \Lambda)$ function, and $k$ is the $x$-component of the spatial frequency. This is shown in Fig. 3(d) and (e). If the sideband is too close to the $k$-spectrum of the pulse envelope (which is centered at $k=0$ ), $\Theta(k)$ cannot separate the transient grating from the pulse envelope. This necessitates a larger spatial sample and/or finer grating period, considerations that have informed our pump-probe beam geometry.

Even though TG-SSSI is a single-shot method, averaging many shots of a reproducible transient process enables significant enhancement of the signal-to-noise ratio. Before averaging, however, the shot-to-shot shifting of the spatial interference fringes (from mechanical vibrations in the optical setup) must be compensated. The fringes are effectively forced into common alignment by adding a constant phase $\Delta \tilde{\phi}_{n}\left(k_{c}, \tau_{c}\right)$ to each frame, giving

$$
\Delta \bar{\Phi}(x, \tau)=\arg \left(\frac{1}{N} \sum_{n=1}^{N} \mathcal{F}_{k}^{-1}\left\{\left[\Delta \tilde{\phi}_{n}(k, \tau) \times \exp \left(\operatorname{iarg}\left(\Delta \tilde{\phi}_{n}\left(k_{c}, \tau_{c}\right)\right)\right)\right] \Theta(k)\right\}\right),
$$

where $\Delta \tilde{\phi}_{n}(k, \tau)=\mathcal{F}_{x}\left\{\Delta \phi_{n}(x, \tau)\right\}, \Delta \tilde{\phi}_{n}\left(k_{c}, \tau_{c}\right)$ is the constant phase added to frame $n$ to align the fringes, $\left(k_{c}, \tau_{c}\right)$ is a common point across all $N$ frames, and $\Delta \bar{\Phi}(x, \tau)$ is the mean spatiotemporal phase. The point $\left(k_{c}, \tau_{c}\right)$ is chosen at a location in the sideband where the signal is sufficiently larger than the phase noise, otherwise each frame would be offset by a random phase factor. 


\section{Results and Discussion}

To demonstrate TG-SSSI, we used the $4 f$ pulse-shaper to generate (a) a Gaussian pulse ( $l=$ 0 , no phase plate), and STOV-carrying pulses with topological charge (b) $l=+1$, (c) $l=-1$, and (d) $l=-2$, using corresponding spiral phase plates in the Fourier plane of the shaper. The columns of Fig. 4 show $\Delta \phi(x, \tau), I_{s}(x, \tau), f(x, \tau)$, and $\Delta \Phi(x, \tau)$ for pulses carrying $l=0, \pm 1$, and -2 . For $l=0$ (row (a)), we see a slight fringe curvature in the transient grating $f(x, \tau)$, indicating a dispersion mismatch between $E_{S}$ and $\mathcal{E}_{i}$. For the $l= \pm 1$ STOVs in rows (b) and (c), $f(x, \tau)$ clearly shows the transient fringe fusing or splitting identifying the opposite phase windings shown in the $\Delta \Phi(x, \tau)$ column. For $l=-2$ (row (d)), one fringe in $f(x, \tau)$ splits into three at the center of the pulse. Upon phase extraction, $\Delta \Phi(x, \tau)$ has two nearby $l=-1$ phase windings rather than a single $l=-2$ winding. We attribute this to a mismatch between the transverse beam dimensions at the Fourier plane of the pulse shaper and the radially independent phase winding of the $l=-2$ phase plate. Since the profile of the beam in the Fourier plane of the shaper (itself dictated by the grating periodicity and cylindrical lens focal length) is slightly elliptical, one of the axes of the phase plate should ideally be scaled to match the ellipticity of the beam. Utilizing a programmable

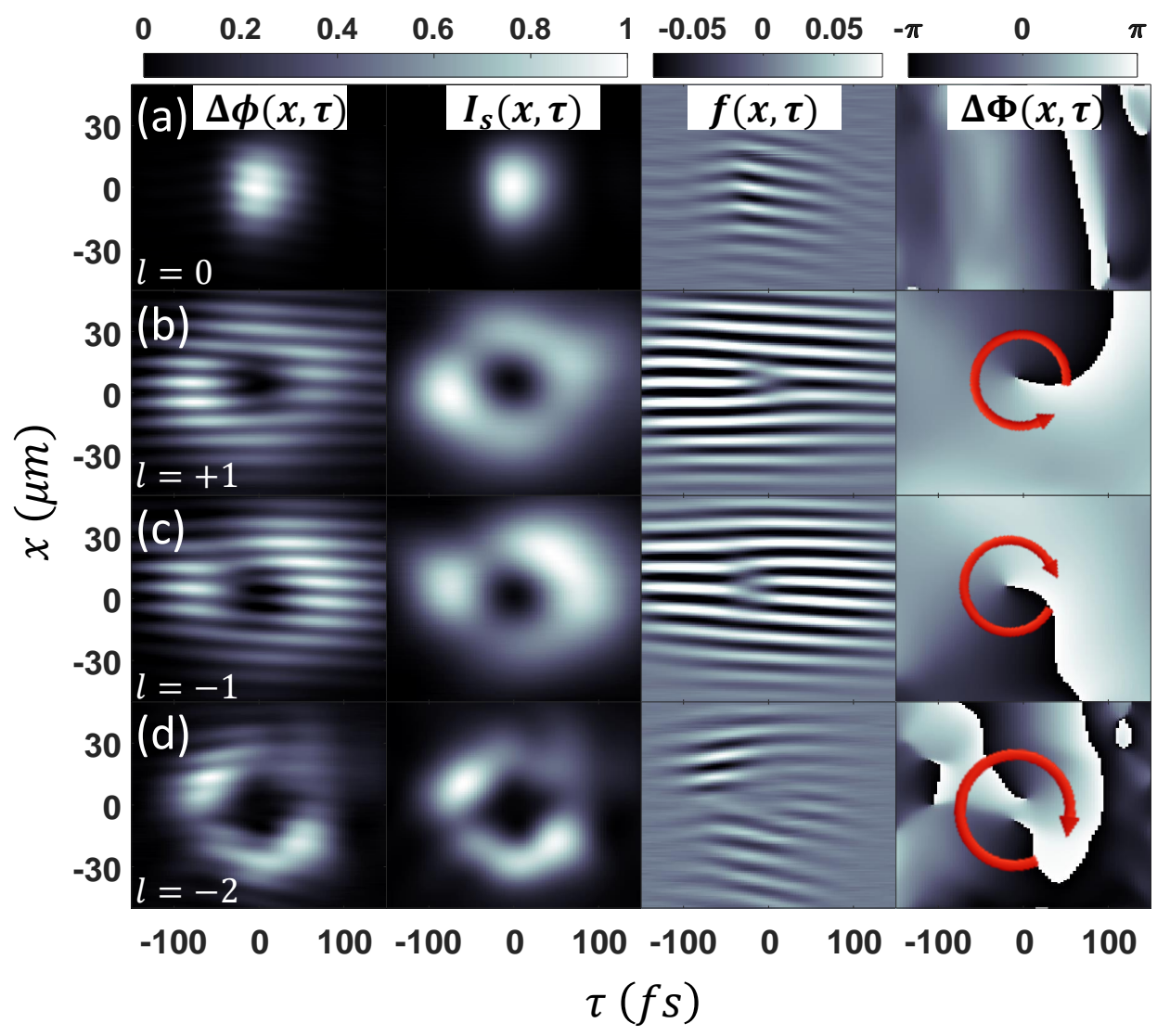

Fig. 4. Experimental results from TG-SSSI after fringe alignment. Columns show the extracted full TG-SSSI signal $\Delta \phi(x, \tau)$, which is low-pass filtered to yield the pulse intensity envelope $I_{S}(x, \tau)$, or high-pass filtered to give the transient grating $f(x, \tau)$, from which the spatiotemporal phase $\Delta \Phi(x, \tau)$ is extracted. The rows show results for (a) a Gaussian pulse $(l=0)$, (b) a $l=+1 \mathrm{STOV}$, (b) a $l=-1 \mathrm{STOV}$, and (c) results from an $l=-2$ phase plate. The red arrows denote the direction of increasing spatiotemporal phase. 
spatial light modulator rather than a fixed phase plate in the pulse shaper would enable scaling of the phase mask to match the beam profile, making possible the generation of $l= \pm 2$ and even higher order STOVs.

\section{Conclusion}

In summary, we have presented a new single-shot diagnostic of ultrashort spatio-temporally structured laser pulses, transient grating single-shot supercontinuum spectral interferometry (TGSSSI) and have used it to measure simple Gaussian and STOV-carrying pulses generated by a $4 f$ pulse-shaper. Among multiple possible applications, TG-SSSI should prove useful in the study of nonlinear propagation, collapse and collapse arrest of intense laser pulses in transparent media, where spatio-temporal optical pulse structures naturally emerge [25]. Finally, we note for experiments where the structured pulse $E_{S}$ is highly repetitive and reproducible, TG-SSSI could be extended to two spatial dimensions ( $x$ and $y$ ) by transversely scanning $E_{p r}^{o u t}$ across the spectrometer entrance slit in the $y$ direction, as is done in $2 \mathrm{D}+1 \operatorname{SSSI}[20]$, to obtain $I_{S}(x, y, \tau)$ and $\Delta \Phi(x, y, \tau)$.

Acknowledgements The authors thank I. Larkin for discussions and technical assistance. This work is supported by Air Force Office of Scientific Research (FA9550-16-10121, FA9550-1610284); Office of Naval Research (N00014-17-1-2705, N00014-20-1-2233); National Science Foundation (PHY2010511).

\section{References}

1. F. Salin, P. Georges, G. Roger, and A. Brun, "Single-shot measurement of a 52-fs pulse," Appl. Opt. 26, 45284531 (1987).

2. R. Trebino, and D. J. Kane, "Using phase retrieval to measure the intensity and phase of ultrashort pulses: frequency-resolved optical gating," J. Opt. Soc. Am. A. 10, 1101-1111 (1993).

3. D. J. Kane, and R. Trebino, "Single-shot measurement of the intensity and phase of an arbitrary ultrashort pulse by using frequency-resolved optical gating," Opt. Lett. 18, 823-825 (1993).

4. J. N. Sweetser, D. N. Fittinghoff, and R. Trebino, "Transient-grating frequency-resolved optical gating," Opt. Lett. 22, 519-521 (1997).

5. K. W. Delong, R. Trebino, J. Hunter, and W. E. White, "Frequency-resolved optical gating with the use of second-harmonic generation," J. Opt. Soc. Am. B. 11, 2206-2215 (1994).

6. P. O'Shea, M. Kimmel, X. Gu, and R. Trebino, "Highly simplified device for ultrashort-pulse measurement," Opt. Lett. 26, 932-934 (2001).

7. C. Iaconis and I. A. Walmsley, "Spectral phase interferometry for direct electric-field reconstruction of ultrashort optical pulses," Opt. Lett. 23, $792-794$ (1998).

8. P. Baum, S. Lochbrunner, and E. Riedle, "Zero-additional-phase SPIDER: full characterization of visible and sub-20-fs ultraviolet pulses," Opt. Lett. 29, 210-212 (2004).

9. M. Hirasawa, N. Nakagawa, K. Yamamoto, R. Morita, H. Shigekawa, and M. Yamashita, "Sensitivity improvement of spectral phase interferometry for direct electric-field reconstruction for the characterization of low-intensity femtosecond pulses," Appl. Phys. B. 74, s225-s229 (2002).

10. C. Dorrer, P. Londero, and I. A. Walmsley, "Homodyne detection in spectral phase interferometry for direct electric-field reconstruction," Opt. Lett. 26, 1510-1512 (2001).

11. A. Monmaryrant, M. Joffre, T. Oksenhendler, R. Herzog, D. Kaplan, and P. Tournois, “Time-domain interferometry for direct electric-field reconstruction by use of an acousto-optic programmable filter and a twophoton detector," Opt. Lett. 28, 278-280 (2003).

12. E. M. Kosik, A. S. Radunsky, I. A. Walmsley, and C. Dorrer, "Interferometric technique for measuring broadband ultrashort pulses at the sampling limit," Opt. Lett. 30, 326-328 (2005).

13. K. Y. Kim, I. Alexeev, and H. M. Milchberg, "Single-shot supercontinuum spectral interferometry," Appl. Phys. Lett. 81, 4124-4126 (2002). 
14. J. K. Wahlstrand, S. Zahedpour, and H. M. Milchberg, "Optimizing the time resolution of supercontinuum spectral interferometry," J. Opt. Soc. Am. B 33, 1476-1481 (2016).

15. D. Patel, D. Jang, S. W. Hancock, H. M. Milchberg, and K. Y. Kim, "Simplified single-shot supercontinuum spectral interferometry," Optics Express 28, 11023-11032 (2020).

16. Z. Guang, M. Rhodes, M. Davis, and R. Trebino, "Complete characterization of a spatiotemporally complex pulse by an improved single-frame pulse-measurement technique," J. Opt. Soc. Am. B 31, 2736-2743 (2014).

17. M. Louisy, C. Guo, L. Neoričić, S. Zhong, A. L'Huillier, C. L. Arnold, and M. Miranda, "Compact single-shot d-scan setup for the characterization of few-cycle laser pulses," Appl. Opt. 56, 9084-9089 (2017).

18. S. P. Le Blanc, E. W. Gaul, N. H. Matlis, A. Rundquist, and M. C. Downer, "Single-shot measurement of temporal phase shifts by frequency-domain holography," Opt. Lett. 25, 764-766 (2000).

19. J. P. Geindre, P. Audebert, A. Rousse, F. Falliès, J. C. Gauthier, A. Mysyrowicz, A. Dos Santos, G. Hamoniaux, and A. Antonetti, "Frequency-domain interferometer for measuring the phase and amplitude of a femtosecond pulse probing a laser-produced plasma," Opt. Lett. 23, 1997-1999 (1994).

20. J. K. Wahlstrand, S. Zahedpour, A. Bahl, M. Kolesik, and H. M. Milchberg, "Bound-electron nonlinearity beyond the ionization threshold," Phys. Rev. Lett. 120, 183901 (2018).

21. Y. H. Chen, S. Varma, A. York, and H. M. Milchberg, "Single-shot, space- and time-resolved measurement of rotational wavepacket revivals in $\mathrm{H}_{2}, \mathrm{D}_{2}, \mathrm{~N}_{2}, \mathrm{O}_{2}$, and $\mathrm{N}_{2} \mathrm{O}$," Opt. Exp. 15, 11341-11357 (2007).

22. J. K. Wahlstrand, S. Zahedpour, Y.-H. Cheng, J. P. Palastro, and H. M. Milchberg, "Absolute measurement of the ultrafast nonlinear electronic and rovibrational response in $\mathrm{H}_{2}$ and $\mathrm{D}_{2}$," Phys. Rev. A 92, 063828 (2015).

23. S. Zahedpour, S. W. Hancock, and H. M. Milchberg, "Ultrashort infrared 2.5-11um pulses: spatiotemporal profiles and absolute nonlinear response of air constinuents," Opt. Lett. 44, 843-846 (2019).

24. S. W. Hancock, S. Zahedpour, A. Goffin, and H. M. Milchberg, "Free-space propagation of spatiotemporal optical vortices," Optica 6, 1547-1553 (2019).

25. N. Jhajj, I. Larkin, E. W. Rosenthal, S. Zahedpour, J. K. Wahlstrand, and H. M. Milchberg, "Spatiotemporal Optical Vortices,” Phys. Rev. X 6, 031037 (2016).

26. L. Allen, M. W. Beijersbergen, R. J. C. Spreeuw, and J. P. Woerdman, "Orbital angular momentum of light and the transformation of Laguerre-Gaussian laser modes," Phys. Rev. A. 45, 8185 (1992).

27. M. A. Porras, "Upper Bound to the Orbital Angular Momentum Carried by an Ultrashort Pulse," Phys. Rev. Lett. 122, 123904 (2019).

28. L. Marrucci, C. Manzo, and D. Paparo, "Optical Spin-to-Orbit Angular Momentum Conversion in Inhomogeneous Anisotropic Media,” Phys. Rev. Lett. 96, 163905 (2006).

29. S. Carbajo, E. Granados, D. Schimpf, A. Sell, K.-H. Hong, J. Moses, and F. X. Kärtner, "Efficient generation of ultra-intense few-cycle radially polarized laser pulses," Opt. Lett. 39, 2487-2490 (2014).

30. J. P. Heritage, R. N. Thurston, W. J. Tomlinson, A. M. Weiner, and R. H. Stolen, "Spectral windowing of frequency-modulated optical pulses in a grating compressor," Appl. Phys. Lett. 47, 87-89 (1985).

31. A. M. Weiner, J. P. Heritage, and E. M. Kirschner, "High-resolution femtosecond pulse shaping," J. Opt. Soc. Am. B. 5, 1563-1572 (1988).

32. A. M. Weiner, and D. E. Leaird, "Generation of terahertz-rate trains of femtosecond pulses by phase-only filtering," Opt. Lett. 15, 51-53 (1990).

33. N. Jhajj, "Hydrodynamic and electrodynamic implications of optical femtosecond filamentation," Ph.D. dissertation (University of Maryland, 2017), http://drum.lib.umd.edu/handle/1903/19973 , Chap. 5.

34. S. Zahedpour, S. W. Hancock, and H. M. Milchberg, "Direct measurement of linearly imposed spatiotemporal optical vortices (STOVs)," in Frontiers in Optics + Laser Science APS/DLS, OSA Technical Digest (Optical Society of America, 2019), paper FW5F.5.

35. W. R. Klein and B. D. Cook, "Unified Approach to Ultrasonic Light Diffraction," IEEE Trans. Sonics Ultrason. 14, 123-134 (1967).

36. A. Couairon, E. Brambilla, T. Corti, D. Majus, O. de J. Ramırez-Gongora, and M. Kolesik, “Practitioner's guide to laser pulse propagation models and simulation,” Eur. Phys. J. Special Topics 199, 5 (2011).

37. M. Kolesik and J. V. Moloney, "Nonlinear optical pulse propagation simulation: From Maxwell's to unidirectional equations," Phys. Rev. E. 70, 036604 (2004). 